\title{
BMJ Open Protocol for an observational study to identify potential predictors of an acute exacerbation in patients with chronic obstructive pulmonary disease (the PACE Study)
}

\author{
Klaus Kenn, ${ }^{1,2}$ Rainer Gloeckl (D) ,1,2 Daniela Leitl, ${ }^{1,2}$ Tessa Schneeberger, ${ }^{1,2}$ \\ Inga Jarosch, ${ }^{1,2}$ Wolfgang Hitzl, ${ }^{3,4,5}$ Peter Alter, ${ }^{6}$ Bernd Sczepanski, ${ }^{2}$ \\ Sandra Winterkamp, ${ }^{2}$ Martina Boensch, ${ }^{2}$ Carmen Schade-Brittinger, ${ }^{7}$ \\ Chrysanthi Skevaki, ${ }^{8}$ Olaf Holz, ${ }^{9}$ Paul W Jones, ${ }^{10}$ Claus F Vogelmeier, ${ }^{6}$ \\ Andreas R Koczulla ${ }^{1,2,11}$
}

To cite: Kenn K, Gloeckl R, Leitl D, et al. Protocol for an observational study to identify potential predictors of an acute exacerbation in patients with chronic obstructive pulmonary disease (the PACE Study). BMJ Open 2021;11:e043014. doi:10.1136/ bmjopen-2020-043014

- Prepublication history for this paper is available online. To view these files, please visit the journal online (http://dx.doi. org/10.1136/bmjopen-2020043014).

Received 03 August 2020 Revised 21 December 2020 Accepted 20 January 2021

Check for updates

(c) Author(s) (or their employer(s)) 2021. Re-use permitted under CC BY-NC. No commercial re-use. See rights and permissions. Published by BMJ.

For numbered affiliations see end of article.

Correspondence to Professor Klaus Kenn; kkenn@schoen-klinik.de

\section{ABSTRACT}

Introduction Acute exacerbations of chronic obstructive pulmonary disease (AECOPD) are the most critical events for patients with COPD that have a negative impact on patients' quality of life, accelerate disease progression, and can result in hospital admissions and death. Although there is no distinct definition or detailed knowledge about AECOPD, it is commonly used as primary outcome in clinical studies. Furthermore, it may be difficult in clinical practice to differentiate the worsening of symptoms due to an AECOPD or to the development of heart failure. Therefore, it is of major clinical importance to investigate the underlying pathophysiology, and if possible, predictors of an AECOPD and thus to identify patients who are at high risk for developing an acute exacerbation.

Methods and analysis In total, 355 patients with COPD will be included prospectively to this study during a 3-week inpatient pulmonary rehabilitation programme at the Schoen Klinik Berchtesgadener Land, Schoenau am Koenigssee (Germany). All patients will be closely monitored from admission to discharge. Lung function, exercise tests, clinical parameters, quality of life, physical activity and symptoms will be recorded, and blood samples and exhaled air will be collected. If a patient develops an AECOPD, there will be additional comprehensive diagnostic assessments to differentiate between cardiac, pulmonary or cardiopulmonary causes of worsening. Follow-up measures will be performed at 6,12 and 24 months. Exploratory data analyses methods will be used for the primary research question (screening and identification of possible factors to predict an AECOPD). Regression analyses and a generalised linear model with a binomial outcome (AECOPD) will be applied to test if predictors are significant.

Ethics and dissemination This study has been approved by the Ethical Committee of the Philipps University Marburg, Germany (№. 61/19). The results will be presented in conferences and published in a peerreviewed journal.

Trial registration number NCT04140097.
Strengths and limitations of this study

- To the best of our knowledge, this is the largest chronic obstructive pulmonary disease (COPD) cohort with prospectively collected data to investigate the development of an acute exacerbation.

- A large variety of assessments including basic science approaches will be used to detect potential early predictors for an acute exacerbation.

- Acute exacerbation diagnosis will be based on clinical signs and judged by an experienced pulmonologist.

- This is the first study to differentiate between COPD-related exacerbation and cardiac-related exacerbation.

- This study is based on exploratory data analyses methods without a sample size or power computation.

\section{INTRODUCTION}

Chronic obstructive pulmonary disease $(\mathrm{COPD})^{1}$ is a lung disease characterised by respiratory problems and poor airflow with dyspnoea and cough being the main symptoms leading to impaired exercise performance and quality of life. $^{2}$ COPD is a progressive disease that typically worsens over time. ${ }^{2}$ In this context, acute exacerbations of COPD (AECOPD) are the most critical events for patients with COPD, essential for the clinical course and the prognosis. The American Thoracic Society and the European Respiratory Society define AECOPD clinically as an episode of increased respiratory symptoms, particularly dyspnoea, cough, sputum production and increased sputum purulence. ${ }^{3}$ These issues are also reflected in the Global Initiative for Chronic Obstructive Lung Disease (GOLD) 
strategy, which defines an exacerbation as 'an acute worsening of respiratory symptoms that results in additional therapy'? It is assumed that the majority of AECOPD are triggered by infections mainly with respiratory viruses ${ }^{4}$ or pathogenic bacteria ${ }^{5}$ which increase local and systemic inflammation. ${ }^{6}$ Exacerbations have a negative impact on the quality of life of patients with $\mathrm{COPD},{ }^{7}$ accelerate disease progression, and can result in hospital admissions and death. ${ }^{89}$ Also within healthcare systems significant variability exists between physicians in both diagnostic practices and prescribing habits at exacerbations, ${ }^{10}$ and the utility of biomarkers to guide clinicians in exacerbation diagnosis and treatment is currently limited. ${ }^{11} 12$

Furthermore, physical comorbidities, such as cardiovascular disease, are also often prevalent in patients with $\mathrm{COPD}^{13}$ and are an additional challenge that impacts clinicians' decision-making at COPD exacerbation. ${ }^{14}$ In patients with COPD with concomitant cardiovascular disease, exacerbations confer an increased risk of subsequent cardiovascular disease events, especially in hospitalised patients and within the first 30 days post-exacerbation. ${ }^{15}$

Until now, research in the field of AECOPD has gathered and analysed data usually after manifestation of AECOPD until recovery and most of them used a retrospective study design. ${ }^{16}{ }^{17}$ A recent systematic review on the monitoring of physiological parameters (eg, oxygen saturation, heart rate or respiratory rate) to predict an AECOPD concluded that there is currently insufficient information on how physiological parameters vary prior to exacerbation to support routine domiciliary monitoring for the prediction of AECOPD. ${ }^{18}$ It would be of extreme clinical importance to determine early predictors of an AECOPD and to identify patients who are at high risk for developing an acute exacerbation and/or to detect the beginning of or prevent an acute exacerbation as early as possible. Early predictors of AECOPD could help to develop novel therapeutic treatment options and strategies, to prevent acute exacerbations in the future for patients with COPD.

\section{METHODS AND ANALYSIS}

\section{Study design and registration}

This study will be a monocentric, prospective observational study. Patients will be recruited during an inpatient pulmonary rehabilitation programme at the reference centre of the Philipps University Marburg: Schoen Klinik Berchtesgadener Land at Schoenau am Koenigssee (Germany). From an own former retrospective analysis in 401 patients with very severe COPD, we learnt that $15.2 \%$ of these patients develop an AECOPD during their stay in the clinic. ${ }^{19}$ Therefore, the setting of an inpatient pulmonary rehabilitation offers a unique model to study the phenomenon of an AECOPD prior to the first visible clinical sign and further on to have a follow-up of the underlying mechanisms. All patients with COPD who are referred to the reference centre for a rehabilitation programme will be included after providing written informed consent. Prospectively, a wide range of measurements will be collected (figure 1). If a patient develops an AECOPD, there will be an additional comprehensive diagnostic assessment to differentiate between pulmonary, cardiac or cardiopulmonary reasons for the acute exacerbation (table 1). The diagnosis of an AECOPD will be made clinically by an experienced pulmonologist within the first 24 hours according to the Anthonisen criteria with the occurrence of at least two symptoms among sputum volume, sputum purulence and breathlessness. ${ }^{20}$

\section{Study objectives}

\section{Primary endpoint}

To prospectively identify potential early, respiratory and or cardiac clinical predictors, laboratory parameters, breath print analyses and/or changes in lung function, symptoms and physical activity prior to an AECOPD in patients with COPD.

\section{Secondary endpoint(s)}

- To identify potential mechanisms explaining the nature and composition of an acute exacerbation in patients with COPD.

- To identify and differentiate patterns of acute COPD exacerbation versus cardiac failure in patients with COPD.

- To investigate the potential role of exhaled breath volatile organic compound (VOC) profiles for the early detection of an AECOPD ('COPD pre-exacerbation breath print').

- To use an electronic nose to capture exhaled breath metabolomic profiles for the early detection of an acute exacerbation in patients with COPD ('COPD pre-exacerbation breath print').

- To identify parameters influencing mortality and other adverse events in the total study cohort at 6,12 and 24 months' follow-up.

\section{Recruitment period}

The study started with the first-patient-in in February 2020 and is scheduled for last-patient-out by the end of 2023 (followed by the 6, 12 and 24 months' follow-up measures).

\section{Eligibility criteria}

Inclusion criteria: patients referred to an inpatient pulmonary rehabilitation programme at the Schoen Klinik Berchtesgadener Land (Germany) with a confirmed diagnosis of COPD according to GOLD stage II-IV, ${ }^{2}$ the ability to follow the rehabilitation protocol and a provided written informed consent.

Exclusion criteria: patients with an ongoing AECOPD or a current primary diagnosis of asthma will not be included in the study.

\section{Study measures and outcomes}

Baseline characteristics

The following baseline characters will be recorded at study baseline concerning:

- Age, gender, anthropometrics.

- Regular drug treatment. 


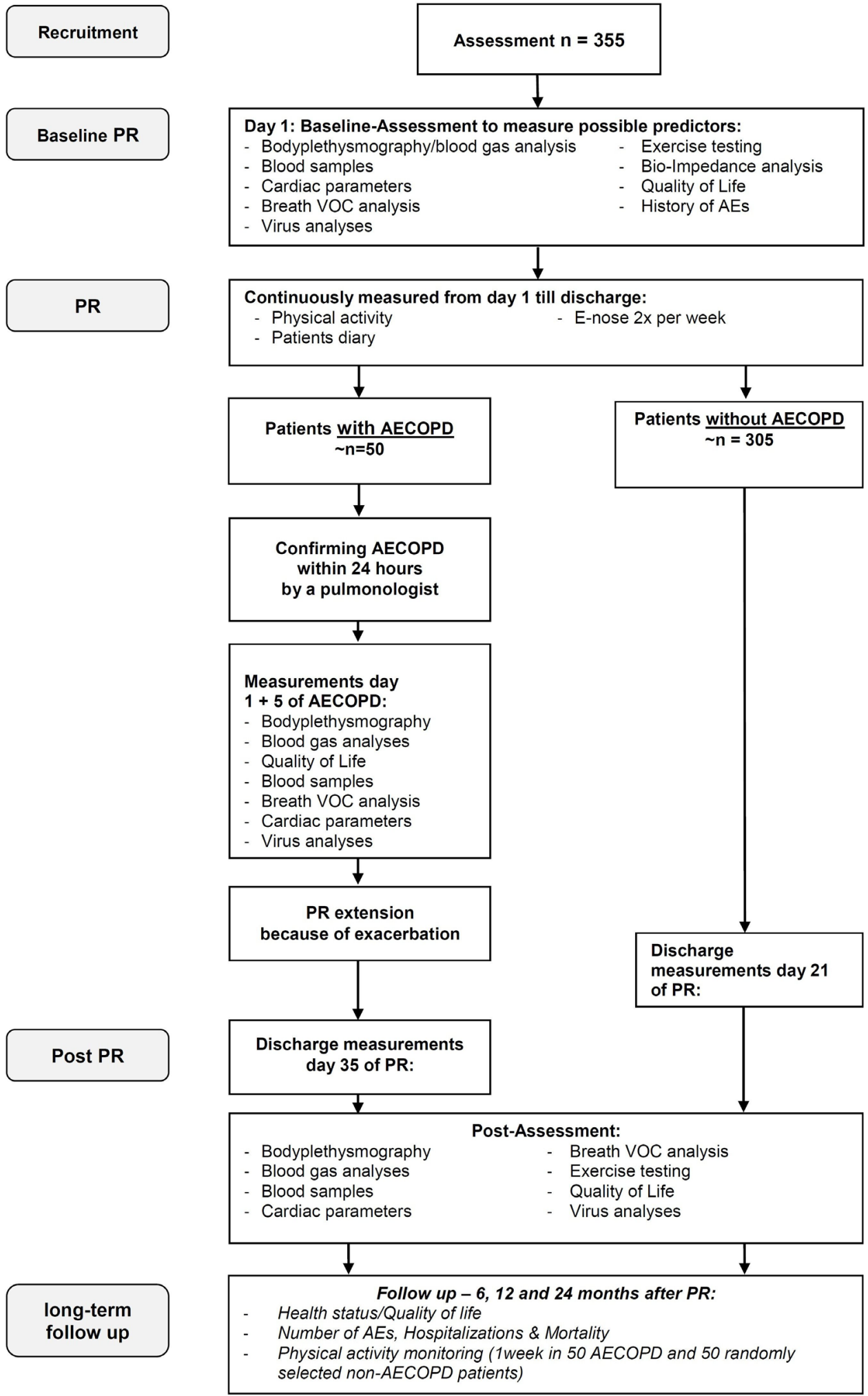

Figure 1 Study flow chart. AECOPD, acute exacerbation of chronic obstructive pulmonary disease; AEs, adverse events; PR, pulmonary rehabilitation; VOC, volatile organic compound.

Social status (family, children), education (years of school and job training), employment status (fulltime, half-time, pension).

- Smoking status (active smoker, ex-smoker, never smoker)
- Number of pack years (1 pack year=1 daily smoked pack of cigarettes for 1 year).

- Long-term oxygen therapy (yes/no, daily usage duration and prescription since when).

- Frailty status according to Fried Frailty Index. ${ }^{21}$ 
Table 1 Overview on study measures

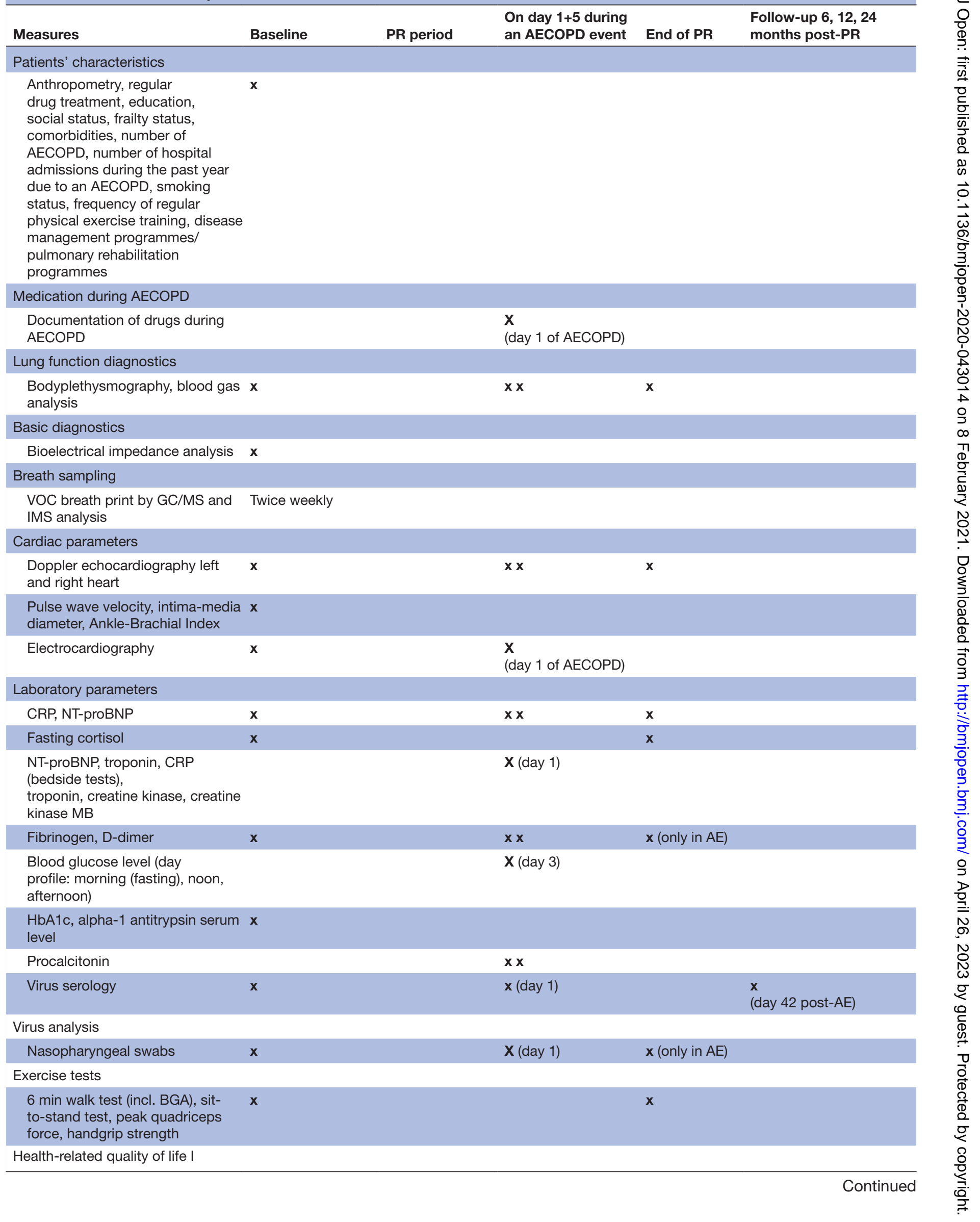


Table 1 Continued

\begin{tabular}{|c|c|c|c|c|c|}
\hline Measures & Baseline & PR period & $\begin{array}{l}\text { On day } 1+5 \text { during } \\
\text { an AECOPD event }\end{array}$ & End of PR & $\begin{array}{l}\text { Follow-up } 6,12,24 \\
\text { months post-PR }\end{array}$ \\
\hline COPD Assessment Test & $\mathbf{x}$ & & $\mathbf{x x}$ & $x$ & $\mathbf{x}$ \\
\hline $\begin{array}{l}36 \text {-item Short Form Health } \\
\text { Survey, mMRC score, COPD } \\
\text { Angst Fragebogen, PHQ-9 }\end{array}$ & $\mathbf{x}$ & & & $\mathbf{x}$ & $\mathbf{x}$ \\
\hline
\end{tabular}

Self-reported daily patients' diary (EXACT)

Daily rating of symptoms

Physical activity monitoring (ActiGraph)

\begin{tabular}{|c|c|c|}
\hline $\begin{array}{l}\text { Continuous monitoring via activity } \\
\text { tracker plus asking for frequency } \\
\text { of regular physical exercise } \\
\text { training }\end{array}$ & $x$ & $\begin{array}{l}\mathbf{x} \\
(1 \text { week only in AECOPD } \\
\text { plus } n=50 \text { non-AECOPD) }\end{array}$ \\
\hline
\end{tabular}

Telephone interview $(6,12,24$

months)

\section{Quality of life I and II, \\ hospitalisations, mortality}

$\mathrm{AE}$, adverse event; $\mathrm{AECOPD}$, acute exacerbation of chronic obstructive pulmonary disease; $\mathrm{BGA}$, blood gas analysis; CRP, $\mathrm{C}$ reactive protein; EXACT, EXAcerbations of Chronic pulmonary disease Tool; GC/MS, gas chromatography/mass spectrometry; HbA1c, haemoglobin A1c; IMS, ion-mobility spectrometry; mMRC, Modified Medical Research Council; NT-proBNP, N-terminal probrain natriuretic peptide; PHQ-9, Patient Health Questionnaire-9; PR, pulmonary rehabilitation; VOC, volatile organic compound.

- Comorbidities according to the criteria by Vanfleteren et $a .^{13}$

- Nocturnal non-invasive ventilation (yes/no, daily usage duration and prescription since when).

- Nocturnal transcutaneous partial pressure of carbon dioxide measurement (Sentec Digitall Monitoring System) in case of hypercapnia to record $\mathrm{CO}_{2}$ max, $\left.\mathrm{SpO}_{2} \min \right)$.

- Number of hospitalisations (with reason) and acute exacerbations within the last year and total so far.

- Patient already attended a disease management programme like, for example, COBRA (yes/no).

- Number of previous pulmonary rehabilitation programmes.

- Frequency of regular physical exercise training (content, days per week and duration) and amount of physical activity (using Physical Activity Vital Sign questionnaire.

\section{Bodyplethysmography and blood gas analysis}

Initial measures of bodyplethysmography on day 1 will be performed after medication use. Blood gas analyses will be performed at rest under ambient conditions after at least 10 min of quiet sitting. Samples will be taken from the hyperaemic earlobe.

\section{Bioelectrical impedance analysis}

Body composition (fat-free mass) will be measured using bioelectrical impedance (NutriGuard-M, Data Input GmbH, Germany). ${ }^{22}$

\section{Breath VOC analysis}

Inflammatory phenotyping in patients with chronic airway disease can be made through blood or sputum analysis. ${ }^{23}$ These assessments do not provide results in real time because they require laboratory procedures. Hence, efforts are ongoing to bring biological assessment to the point of care. If properly validated, molecular profiling of exhaled air may provide a non-invasive and rapid alternative for blood and sputum. ${ }^{24}$ VOCs in exhaled air have been associated with inflammatory profiles in asthma ${ }^{2526}$ and COPD. ${ }^{27}$ For VOC analysis 2 , different approaches will be used:

1. Analysis by ion-mobility spectrometry (IMS) (BioScout IMS, B\&S Analytik, Dortmund, Germany) will be performed. The IMS technology is fast and easy to use. Patients exhale directly into the IMS device where only low volumes of exhalation are needed for analysis. IMS has a great potential to proceed to become a point-ofcare tool that produces characteristic disease-related breath profiles. $^{28}$

2. Analysis by gas chromatography/mass spectrometry (GC/MS): we will collect breath samples on adsorption tubes for the analysis by $\mathrm{GC} / \mathrm{MS},{ }^{29}{ }^{30} \mathrm{using}$ the novel ReCIVA sampler system (Owlstone medical, UK). The patients will inhale precleaned pressurised room air and the adsorption tubes will be loaded with $500 \mathrm{~mL}$ of exhaled breath. The tubes will then be shipped to Fraunhofer ITEM (Hannover, Germany) for GC/MS analysis. 
It can be assumed that the clinical change from stable to exacerbated patients with COPD independent of the cause of the exacerbation will change the pattern of exhaled VOCs in patients with COPD. ${ }^{31}$ The early detection of these changes and a respective adjustment of treatment could potentially avoid the number of COPD exacerbations. Based on VOC pattern and clinical characteristics, COPD phenotypes could be defined.

\section{Cardiovascular assessment}

Echocardiography will be performed to assess morphology and function of the left and right heart, including Doppler flow measurements. The intima-media diameter, the pulse wave transit time and the Ankle-Brachial Index will be assessed. In case of an AECOPD, a resting electrocardiography will be performed.

\section{Virus analyses}

Nasopharyngeal swabs will be collected by means of nylon flocked swabs in combination with universal transport medium-RT (Copan Diagnostics, Murrieta, California, USA) and stored at $-80^{\circ} \mathrm{C}$. Viral RNA will be extracted with a QIAamp viral RNA purification kit (Qiagen, Valencia, California, USA) and tested by PCR for the determination of the most common respiratory viruses (including SARS-CoV-2, respiratory syncytial virus, influenza A, influenza B, rhinovirus, human metapneumovirus, adenovirus, human bocavirus). Moreover, whole genome sequencing of the same samples will be processed by next generation sequencing for analyses of the virome.

\section{Venous blood samples}

Basic inflammatory parameters will be analysed directly. Furthermore, blood samples will be frozen at $-80^{\circ} \mathrm{C}$ and stored for further comprehensive analysis on, for example, virus-induced reasons for an AECOPD and a variety of clinically relevant serological parameters being able to modulate inflammatory processes (eg, interleukins, tumour necrosis factor-alpha).

\section{Exercise testing}

- Six-minute walk test (6MWT) including blood gas analyses pre/post-6MWT will be performed in accordance with international recommendations. ${ }^{32}$

- Sit-to-stand tests (STSTs).

STSTs involve either the number of sit-to-stand repetitions from a standard chair within $1 \mathrm{~min}$ (1 min STST), ${ }^{33}$ or quantify the time that a patient needs to perform five repetitions in a row (5-repetition STST).

- Peak quadriceps force.

Isometric peak quadriceps force will be measured at $90^{\circ}$ knee angle using a fixed dynamometer on a kneeextension training device. At least three attempts will be performed with the best value in Newton and \%pred. being recorded. ${ }^{34}$

- Peak handgrip force.

Isometric peak handgrip force will be measured by handheld dynamometry (JAMAR hydraulic hand dynamometer) in a $90^{\circ}$ elbow angle position. At least three attempts will be performed with the best value in kilogram and \% pred. ${ }^{35}$ are recorded.

\section{Quality-of-life measures}

- The 36-item Short Form Health Survey.

Generic health-related quality-of-life (HRQL) measure. The instrument is used widely to evaluate HRQL across various populations. ${ }^{36}$ There are two summary scales: an overall physical and a mental health component summary score.

- COPD Assessment Test (CAT).

The CAT is a patient-completed questionnaire assessing the global impact of COPD on health status with scores $<10$ indicating low, 10-20 medium, 21-30 high and $>30$ very high influence on health status. ${ }^{37}$

- COPD Angst Fragebogen (CAF).

The CAF questionnaire is a reliable and valid questionnaire to determine COPD-specific anxiety according to the following domains: social isolation, dyspnoea, physical activity, disease progression, partnership and long-term oxygen therapy.

- Patient Health Questionnaire-9 (PHQ-9).

The PHQ-9 is a self-administered patient questionnaire that monitors the severity of depression and response to treatment (score: $0-27$ ).

- Modified Medical Research Council score (mMRC score).

The mMRC score is a simple grading system for patients with respiratory diseases, to assess degree of baseline functional disability due to dyspnoea.

\section{Self-reported patient diary (EXAcerbations of Chronic pulmonary} disease Tool-patient-reported outcome)

The EXAcerbations of Chronic pulmonary disease Tool is a 14-item patient-reported outcome daily diary used to quantify and measure exacerbations of COPD. ${ }^{1}$ It provides a simple, standardised approach for assessing the symptomatic manifestations of COPD exacerbations, with a development and validation history consistent with guidelines proposed by the Food and Drug Administration, and well-established measurement principles.

\section{Physical activity monitoring}

Physical activity will be measured using the GT3X+device from ActiGraph, a validated device in COPD. ${ }^{38}$ The device includes both a microelectromechanical system-based accelerometer and an ambient light sensor. Average steps per day as well as time spent in different physical activity levels during the day (low, moderate, vigorous) will be analysed.

\section{Follow-up measures (at 6, 12, 24 months)}

Following outcomes will be reassessed at the follow-up measures: number of hospitalisations, number of AECOPD, quality of life, amount of regular exercise training and physical activity monitoring (in a subgroup). Furthermore, if patients die during the follow-up period, date and cause of death will be recorded. 


\section{Safety evaluation}

The clinic has an established protocol for managing patients if they develop an exacerbation during the programme. This protocol will be followed for the patients admitted to this study. In case of an AECOPD, patients are clinically diagnosed by a pulmonologist and treated according to international guidelines. ${ }^{3}$ In case of a life-threatening deterioration, patients are referred to an acute hospital. Otherwise, the AECOPD treatment is continued during the inpatient pulmonary rehabilitation (PR) programme or at an intermediate care room under close monitoring at the recruitment centre. Adverse events during the PR phase will be recorded in the electronic case report form (e-CRF).

\section{Sample size estimation and recruitment target}

In a previously presented analysis of pretransplant patients with COPD ( $\mathrm{n}=401), 15 \%$ developed an AECOPD during the inpatient rehabilitation period. ${ }^{19}$ Therefore, we aim to include 355 patients with COPD in this study to end up with an adequate cohort of about 50 patients with AECOPD for further exploratory data analyses.

The recruiting reference centre (University of Marburg Teaching Hospital Schoen Klinik Berchtesgadener Land) represents a unique setting for this comprehensive observational study including diagnostic and scientific expertise. In 2018, a total of 300 patients with COPD have been referred to this centre. Due to staff and logistic reasons, only every second patient (according to the running patient ID) will be assessed for eligibility. It is very likely that the estimated sample size can be recruited within 36 months. A refusal rate of about 20\% (95 out of 450 patients who will be assessed for eligibility) is in accordance to our own centre-based study experience with patients with COPD. Also, a dropout rate of about $20 \%$ must be expected (based on study centre experiences). Reasons for dropout will be recorded.

\section{Patient and public involvement}

Prior to the study, we have interviewed several patients with frequent AECOPD in our clinic asking for early signs of AECOPD. We could not elaborate individual predictors for AECOPD. Asking for the importance, patients generally described AECOPD as the most frightening event markedly influencing their quality of life.

The public was not involved in planning this study.

\section{Statistical analyses}

Data will be stored and managed in a password-secured e-CRF (MACRO database) managed by the Coordinating Centre for Clinical Trials of the Philipps University Marburg. Professional data management will also be provided by this institution. Data consistency will be checked and data will be screened for outliers, normality, gamma or lognormal distributions by using quantile plots. Continuous variables will be tested for the abovementioned distributions by using Kolmogrov-Smirnov test. Cross-tabulation tables will be analysed using Fisher's exact test or Pearson's $\mathrm{X}^{2}$ test. Exploratory data analyses methods will be used for the primary research question (screening and identification of possible factors to predict an AECOPD). Univariate correlation (Pearson or Spearman) and regression analyses will be used to restrict the large number of possible predictors in the first step; and then in the second step, this restricted set will be offered to a forward variable selection algorithm to end up with an even smaller set. Finally, this smaller set will be analysed by a generalised linear model with a binomial outcome (AECOPD) which will test if predictors are significant. Secondary research questions will be analysed by using mixed models or generalised estimation equation models which offer modelling of normal, gamma or lognormal distributions. In case of deviations of these distributions, Friedman analysis of variance, Wilcoxon matched pairs test as non-parametric alternatives will be used. It will be clearly stated in the Discussion section of the final manuscript as a limitation of the study that the analysis is based on exploratory data analyses methods without a sample size or power computation. All reported tests will be done two-sided, and $p$ values of $<0.05$ will be considered as statistically significant. All statistical analyses will be done by the use of NCSS (NCSS V.10, NCSS, Kaysville, Utah, USA), STATISTICA V.13 (Hill, T \& Lewicki, P. Statistics: Methods and Applications. StatSoft, Tulsa, Oklahoma, USA).

\section{Ethics and dissemination}

This study has been approved by the Ethical Committee of the Philipps University Marburg, Germany (no. 61/19). There are no safety considerations.

The results of this study will be disseminated by different complimentary strategies:

1. Publication of study results in high-quality international peer-reviewed journals.

2. Presentation and discussion of study results during national and international conferences and congresses.

3. Sharing results with national and international clinical networks and medical societies.

\section{Author affiliations}

${ }^{1}$ Department of Pulmonary Rehabilitation, Member of the German Center for Lung Research (DZL), Philipps University Marburg, Marburg, Germany

${ }^{2}$ Institute for Pulmonary Rehabilitation Research, Schoen Klinik Berchtesgadener Land, Schoenau am Koenigssee, Germany

${ }^{3}$ Research Office (Biostatistics), Paracelsus Medical University Salzburg, Salzburg, Austria

${ }^{4}$ Department of Ophthalmology and Optometry, Paracelsus Medical University Salzburg, Salzburg, Austria

${ }^{5}$ Research Program Experimental Ophtalmology and Glaucoma Reserach, Paracelsus Medical University, Salzburg, Austria

${ }^{6}$ Department of Medicine, Pulmonary and Critical Care Medicine, Member of the German Center for Lung Research (DZL), Philipps University Marburg, Marburg, Germany

${ }^{7}$ Coordinating Centre for Clinical Trials, Member of the German Center for Lung Research (DZL), Philipps University Marburg, Marburg, Germany ${ }^{8}$ Institute of Laboratory Medicine, Universities of Giessen and Marburg Lung Center (UGMLC), Member of the German Center for Lung Research (DZL), Marburg, Germany 
${ }^{9}$ Biomedical Research in Endstage and Obstructive Lung Disease Hannover (BREATH), Fraunhofer Institute for Toxicology and Experimental Medicine (ITEM), Member of the German Center for Lung Research (DZL), Hannover, Germany ${ }^{10}$ Institute of Infection and Immunity, St George's University of London, London, UK ${ }^{11}$ Department of Medicine, Paracelsus Medical University, Salzburg, Austria

Contributors KK, RG, TS, IJ, CFV, PWJ and ARK contributed to the conception and design of the study. WH developed the statistical analysis plan. DL, RG, TS, IJ, SW, $\mathrm{MB}$ and BS were planning clinical study measures and will be involved in collecting data and performing study measures. PA planned all cardiovascular aspects of this study. $\mathrm{OH}$ planned all assessments on collecting breath volatile organic compounds and will perform all analyses. CS-B contributed to the study logistic and the creation of the electronic case report form. CS designed all aspects concerning virus analyses. RG drafted the manuscript. All authors revised the content and approved the final manuscript before publication.

Funding This study will be partly funded by GlaxoSmithKline GmbH \& Co (Munich, Germany). For CS: supported by the Universities Giessen and Marburg Lung Centre (UGMLC), the German Centre for Lung Research (DZL), University Hospital Giessen and Marburg (UKGM) research funding according to article 2, section 3 cooperation agreement, and the Deutsche Forschungsgemeinschaft (DFG)-funded SFB 1021 (C04), KF0 309 (P10), and SK 317/1-1 (project number 428518790) as well as by the Foundation for Pathobiochemistry and Molecular Diagnostics.

Disclaimer The funding body was not involved in developing the study design nor will it be involved in data collection, analyses/interpretation of data or writing the manuscript.

Competing interests For CS: consultancy and research funding, Hycor Biomedical, Bencard Allergie and Thermo Fisher Scientific; research funding, Mead Johnson Nutrition (MJN)

Patient and public involvement Patients and/or the public were involved in the design, or conduct, or reporting, or dissemination plans of this research. Refer to the Methods section for further details.

Patient consent for publication Not required.

Provenance and peer review Not commissioned; externally peer reviewed.

Open access This is an open access article distributed in accordance with the Creative Commons Attribution Non Commercial (CC BY-NC 4.0) license, which permits others to distribute, remix, adapt, build upon this work non-commercially, and license their derivative works on different terms, provided the original work is properly cited, appropriate credit is given, any changes made indicated, and the use is non-commercial. See: http://creativecommons.org/licenses/by-nc/4.0/.

ORCID iD

Rainer Gloeckl http://orcid.org/0000-0002-2741-2748

\section{REFERENCES}

1 Global Initiative for Chronic Obstructive Lung Disease (GOLD). Global strategy for the diagnosis MaPoC, 2015. Available: http://www. goldcopd.org/

2 GOLD-Report. Global strategy of the diagnosis, management, and prevention of chronic obstructive pulmonary disease, 2020. Available: www.goldcopd.org

3 Wedzicha JA, Miravitlles M, Hurst JR, et al. Management of COPD exacerbations: a European Respiratory Society/American Thoracic Society guideline. Eur Respir J 2017;49:1600791.

4 Seemungal T, Harper-Owen R, Bhowmik A, et al. Respiratory viruses, symptoms, and inflammatory markers in acute exacerbations and stable chronic obstructive pulmonary disease. Am J Respir Crit Care Med 2001;164:1618-23.

5 Garcha DS, Thurston SJ, Patel ARC, et al. Changes in prevalence and load of airway bacteria using quantitative PCR in stable and exacerbated COPD. Thorax 2012;67:1075-80.

6 Bafadhel M, Greening NJ, Harvey-Dunstan TC, et al. Blood eosinophils and outcomes in severe hospitalized exacerbations of COPD. Chest 2016;150:320-8.

7 Seemungal TA, Donaldson GC, Paul EA, et al. Effect of exacerbation on quality of life in patients with chronic obstructive pulmonary disease. Am J Respir Crit Care Med 1998;157:1418-22.

8 Burrows B, Bloom JW, Traver GA, et al. The course and prognosis of different forms of chronic airways obstruction in a sample from the general population. N Engl J Med 1987;317:1309-14.
9 Donaldson GC, Seemungal TAR, Bhowmik A, et al. Relationship between exacerbation frequency and lung function decline in chronic obstructive pulmonary disease. Thorax 2002;57:847-52.

10 Boggon R, Hubbard R, Smeeth L, et al. Variability of antibiotic prescribing in patients with chronic obstructive pulmonary disease exacerbations: a cohort study. BMC Pulm Med 2013;13:32.

11 Hurst JR, Donaldson GC, Perera WR, et al. Use of plasma biomarkers at exacerbation of chronic obstructive pulmonary disease. Am J Respir Crit Care Med 2006;174:867-74.

12 Bafadhel M, McKenna S, Terry S, et al. Blood eosinophils to direct corticosteroid treatment of exacerbations of chronic obstructive pulmonary disease: a randomized placebo-controlled trial. $A m \mathrm{~J}$ Respir Crit Care Med 2012;186:48-55.

13 Vanfleteren LEGW, Spruit MA, Groenen M, et al. Clusters of comorbidities based on validated objective measurements and systemic inflammation in patients with chronic obstructive pulmonary disease. Am J Respir Crit Care Med 2013;187:728-35.

14 Risør MB, Spigt M, Iversen R, et al. The complexity of managing COPD exacerbations: a grounded theory study of European general practice. BMJ Open 2013;3:e003861.

15 Kunisaki KM, Dransfield MT, Anderson JA. Exacerbations of chronic obstructive pulmonary disease and cardiac events: a cohort analysis. Am J Respir Crit Care Med 2018.

16 Cardoso J, Coelho R, Rocha C, et al. Prediction of severe exacerbations and mortality in COPD: the role of exacerbation history and inspiratory capacity/total lung capacity ratio. Int J Chron Obstruct Pulmon Dis 2018;13:1105-13.

17 Stanford RH, Nag A, Mapel DW, et al. Claims-based risk model for first severe COPD exacerbation. Am J Manag Care 2018;24:e45-53.

18 Al Rajeh A, Hurst J. Monitoring of physiological parameters to predict exacerbations of chronic obstructive pulmonary disease (COPD): a systematic review. J Clin Med 2016;5:jcm5120108:108. doi:10.3390/ jcm5120108

19 Heinzelmann I, Ningel I, Gloeckl R. Influence of an acute exacerbation during pulmonary rehabilitation (PR) on outcomes of PR in COPD patients awaiting lung transplantation. Eur Respir $J$ 2014;44:P3045.

20 Anthonisen NR, Manfreda J, Warren CP, et al. Antibiotic therapy in exacerbations of chronic obstructive pulmonary disease. Ann Intern Med 1987;106:196-204.

21 Fried LP, Tangen CM, Walston J, et al. Frailty in older adults: evidence for a phenotype. J Gerontol A Biol Sci Med Sci 2001;56:M146-57.

22 Kyle UG, Bosaeus I, De Lorenzo AD, et al. Bioelectrical impedance analysis--part I: review of principles and methods. Clin Nutr 2004;23:1226-43.

23 Gonem S, Raj V, Wardlaw AJ, et al. Phenotyping airways disease: an A to E approach. Clin Exp Allergy 2012;42:1664-83.

24 Bos LD, Sterk PJ, Fowler SJ. Breathomics in the setting of asthma and chronic obstructive pulmonary disease. J Allergy Clin Immunol 2016;138:970-6.

25 Ibrahim B, Basanta M, Cadden P, et al. Non-Invasive phenotyping using exhaled volatile organic compounds in asthma. Thorax 2011;66:804-9.

26 Schleich FN, Zanella D, Stefanuto P-H, et al. Exhaled volatile organic compounds are able to discriminate between neutrophilic and eosinophilic asthma. Am J Respir Crit Care Med 2019;200:444-53.

27 Fens N, de Nijs SB, Peters S, et al. Exhaled air molecular profiling in relation to inflammatory subtype and activity in COPD. Eur Respir J 2011;38:1301-9.

28 Bach J-P, Gold M, Mengel D, et al. Measuring compounds in exhaled air to detect Alzheimer's disease and Parkinson's disease. PLoS One 2015;10:e0132227.

29 Gaida A, Holz O, Nell C, et al. A dual center study to compare breath volatile organic compounds from smokers and non-smokers with and without COPD. J Breath Res 2016;10:026006.

30 Küppers L, Holz O, Schuchardt S, et al. Breath volatile organic compounds of lung transplant recipients with and without chronic lung allograft dysfunction. J Breath Res 2018;12:036023.

31 de Vries R, Dagelet YWF, Spoor P, et al. Clinical and inflammatory phenotyping by breathomics in chronic airway diseases irrespective of the diagnostic label. Eur Respir J 2018;51:13993003.01817-2017 doi:10.1183/13993003.01817-2017

32 Holland AE, Spruit MA, Troosters T, et al. An official European respiratory Society/American thoracic Society technical standard: field walking tests in chronic respiratory disease. Eur Respir $J$ 2014:44:1428-46.

33 Puhan MA, Siebeling L, Zoller M, et al. Simple functiona performance tests and mortality in COPD. Eur Respir $J$ 2013:42:956-63. 
34 Andrews AW, Thomas MW, Bohannon RW. Normative values for isometric muscle force measurements obtained with hand-held dynamometers. Phys Ther 1996;76:248-59.

35 Tveter AT, Dagfinrud H, Moseng T, et al. Health-Related physical fitness measures: reference values and reference equations for use in clinical practice. Arch Phys Med Rehabil 2014;95:1366-73.

36 McHorney CA, Ware JE, Lu JF, et al. The mos 36-item shortform health survey (SF-36): III. tests of data quality, scaling assumptions, and reliability across diverse patient groups. Med Care 1994:32:40-66.

37 Jones PW, Harding G, Berry P, et al. Development and first validation of the COPD assessment test. Eur Respir $J$ 2009;34:648-54.

38 Rabinovich RA, Louvaris Z, Raste Y, et al. Validity of physical activity monitors during daily life in patients with COPD. Eur Respir $J$ 2013;42:1205-15. 